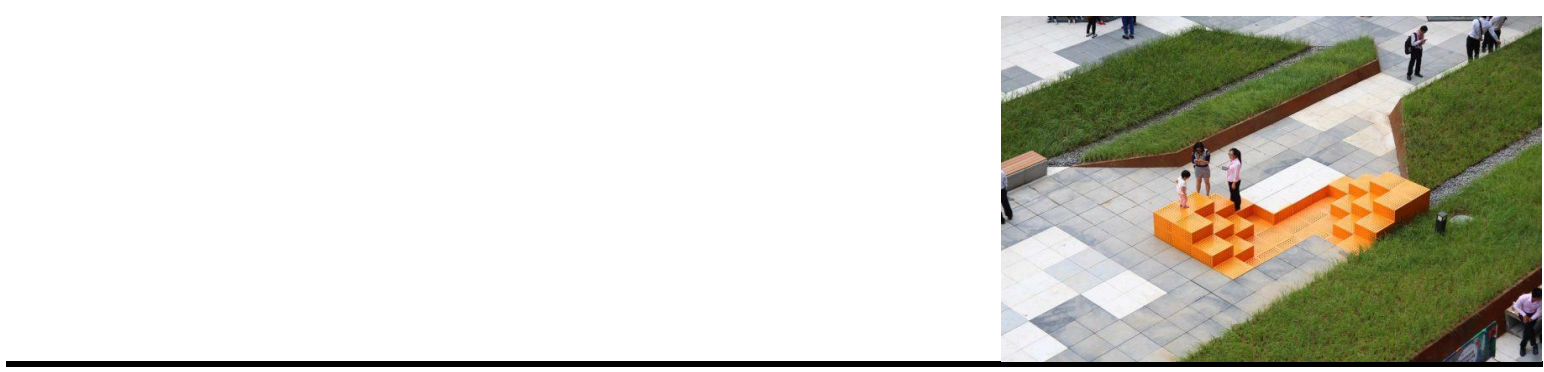

DOI: 10.21005/pif.2020.44.B-04

\title{
UNDERSTANDING THE MEME TERM FROM A GENERAL AND ARCHITECTURAL POINT OF VIEW
}

\author{
Alina Holovatiuk \\ Master of Architecture / PhD student \\ Author's Orcid number: 0000-0003-4705-1027 \\ Kyiv National University of Construction and Architecture, Kyiv, Ukraine \\ Faculty of Architecture \\ Department of Informational technologies in Architecture
}

\section{ABSTRACT}

This article deals with the notion of meme from the general, web and architectural point of view. The history of the creation and the process of further transformation of the term meme, which gradually penetrated from the initial genetic environment into the environment of media and technologies, is described. The disagreements are mentioned both in the interpretation of the concept of a meme and in the definition of a meme as a certain useful or harmful element of culture. By comparing the Internet meme and its main properties with the architectural meme, the characteristics of the last one are indicated.

Key words: meme, symbol, urban public space, architecture, space formation. 


\section{INTRODUCTION}

The concept of "meme" means something intangible (idea, image, concept, association, opinion, style of behavior, phrase, sounds, etc.), in any way (verbally, non-verbally, virtually), transmitted from person to person and that ultimately can become material. The meme is modified by its very bearer, it produces an impact both on him and on society as a whole. The main distinguishing feature of the meme is its ability to create its own copies (replications), in fact, without the existence of copies, a "thing" cannot be called a meme. The second defining criteria is versatility. In some sources, the meme is explained as "a sign, cleared of context," that is, any element of mass culture, after emerging in specific conditions, undergoes replication, gains independence from a specific situation / place and turns into a contextless unit of information. Such a meme can be applied in completely different circumstances, and it will continue to carry its informational content. And the third criteria to consider something a meme is its virality. To fulfill the conditions of this criteria, information must be relevant, draw attention to itself, influence the worldview and cause a desire to convey this unit of information further. Moreover, it is also important who relays the information further - for the success of the campaign, the person-distributor must be of great social importance, be an opinion leader (influencer), or, more simply, have the trust of the masses.

A notion of meme was discussed multiple times from the side of biology, genetics, sociology and psychology, but never - from the architecture point of view.

\section{FORMULATION OF THE PROBLEM}

There are two sciences (although they are not officially recognized) that study the elements of cultural information in one form or another, their causes, meanings, transmission and distribution. The first is called memetics and arose on the basis of genetics, or rather Richard Dawkins' assumption about the human body, that is a mechanism for storing and transmitting not only genetic, but also cultural information. The name of science comes from the Greek word "mimetes", which translates as "imitator" and was later abbreviated by the author himself for convenience. In 1976, Dawkins published "The Selfish Gene", describing the concept of replicators, those so-called "memes", that are complementary to normal sociocultural processes. According to Dawkins, our culture and religion consists of a large number of information units that originated and functioned during specific historical eras, and some have even survived to our time. Moreover, the scientist, supporting the ideas of Charles Darwin, concludes that any life forms exist according to a given rule: all living things evolve as a result of natural selection of small units, self-reproducing themselves, and it can be genes, memes, or even something else unknown.

The idea of copying information is not new at all. For example, the French philosophers of the 20th century Georges Bataille, Gilles Deleuze, explored "simulacra» - copies of the things, that does not exist in reality. "Simulacra" is also not a product of the 20th century - for the first time Plato made a suggestion about them. In his writings they were called "eidolons" and meant an unsuccessful copy, that distorts the prototype idea before implementing in reality. Earlier and simultaneously with Dawkins, other scientists talked about very similar concepts, for example, "culturgenes" (K. Lumsden and E. Wilson) and "mental microbes", however, the ideas did not take root.

In 1994, a new term emerged, the "media virus", which is derived from memes, with the difference, that pieces of information are transmitted through the media and affect the personal judgments of many people (eg, sensations, scandals, gossip, human behavior, etc.). And the "meme" in this theory was seen as a negative phenomenon that has no useful meaning, but causes significant consequences for society. The term was coined by media expert Douglas Rashkoff to describe veiled ideas that are implanted by media viruses in the infosphere and then settle as alien thoughts in the minds of many.

Memes acquire a negative color in the scientific statements of the popularizer of memetics Susan Blackmore. In the early 2000 s, she put forward the theory of meme mutation and the emergence of a completely new species - technological "t-memes". This meme uses human resources ( machines) to evolve faster, copy / reproduce his own kind and, in the end, to flood the entire planet (which, of 
course, is not unambiguously positive for humanity). According to the concept of Blackmore, we do not consciously choose what to copy and what not, so along with useful things / processes / ideas, completely unnecessary, "parasitic" things are replicated. Author includes to these things religion, language and art. For many years of existence side by side with memes-"parasites", we have become accustomed to them and do not notice their selfish nature. The Internet, according to Blackmore, was not created for good purposes either - rather as an environment for faster promotion of information.

Even later, they first talked about memology - a young unrecognized science of the elements of cultural information in the Internet environment. An Internet meme (or Internet phenomenon) is a unit of information, an intangible object that has spontaneously gained popularity and is replicated in digital space by all available means. An online meme, unlike a regular meme, has a shorter life cycle and is much easier to track. Memologists study the patterns and phases of the life of memes, classify and structure them into different categories.

Memology courses have now become a program at many major universities around the world, including Cambridge. In 2018, the first University of Memology appeared in St. Petersburg, where students learn to create memes from news, talk about the taxonomy and morphology of the Internet phenomenon. However, memes have never been directly studied from an architectural point of view and it is worth asking the question: what can be called a meme in architecture and, precisely, in the public spaces of cities?

\section{BASIC THEORY PART}

By analogy with the usual definition, a meme is any physical object of public space, with a message / meaning embedded in it, which is clear at the first acquaintance with it and does not require accompaniment with the necessary explanations. The primary meaning can be also changed and the meme will perform a different function in space. In general, most elements of public space improvement and even groups of these elements can be considered as memes (Fig. 1). From standard sculptures, lanterns, flower beds, benches, fountains, as well as elements of facades that are part of public space or separate public space from each other, to modern kinetic sculptures, bicycle parking lots, ramps, pavilions and even immersive performances, which makes a meme and a certain human activity in public space.

If we emphasize the main characteristics of the Internet meme, in comparison with it it will be possible to better understand the nature of the meme in architecture.

\section{A precedent phenomenon}

Usually, the Internet meme is preceded by the appearance of certain information on the Internet (info drive). It can be related to a significant news / social / political event, touch a famous person, be an element of a movie / book / game or other audio-visual product. That is, the meme always contains information about the source of its origin. Memes arise in the public space of the Internet when the opinions of many people collide and the spontaneous appearance of a successful description / phrase / comment / of the first association to the very precedent of the meme. The event itself often becomes a meme without additional outside interference. This is the first stage of "memization".

In public space, the appearance of any architectural element is preceded by the will of the sponsoring customer. It can also be related to an event or an acute social problem / news (Fig. 2), or it can simply be a power desire, however, the emergence of a new architectural meme is not a spontaneous phenomenon. Internet memes can become popular even in the first minutes of existence because no one owns virtual space (except for closed forums / groups / chats / video streams). This gives more freedom and speed in the dissemination of information and direct reaction to it. Unlike the Internet meme, in reality public space is necessarily in someone's possessions (the state, the city, group of people, semi-private possessions) and if the majority of territories are available for free passage (the open spaces which do not charge a pass), not everyone can make changes (have the authority to establish these changes) in the public space of cities. And, even if the community of a 
certain district has won the right to independent intervention in the local public space, in the physical appearance of any element, as a rule, there is no coincidence. Before the appearance of the physical meme, the priority idea is adjusted to take into account the budget, the material / physical resources involved and a large number of other criteria.

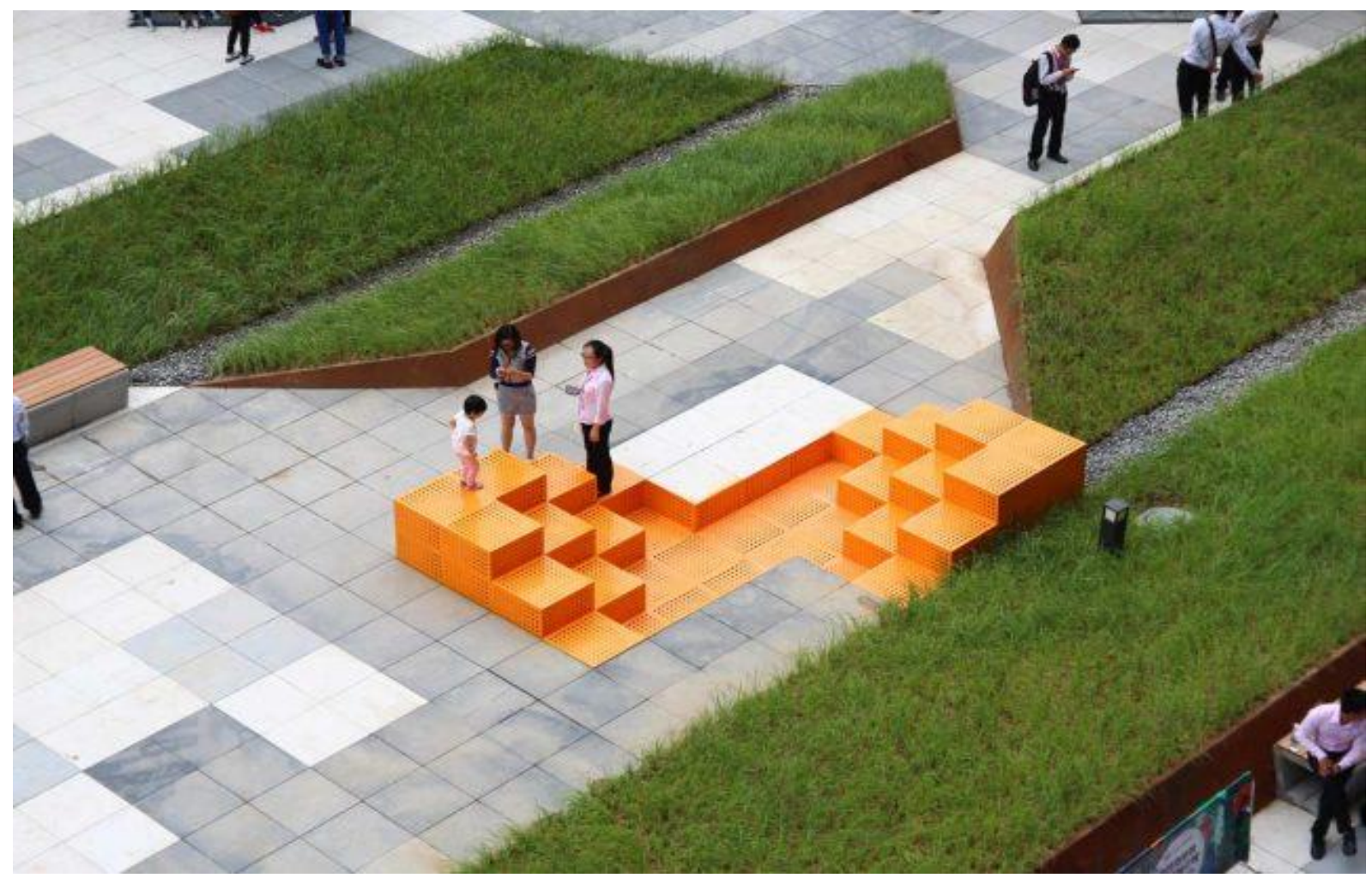

Fig. 1. Vanke Cloud City by Lab D+H. An example of meme. Source: SA Rogers

It is also worth noting that the time interval between the emergence of precedent (desire, order, design, obtaining permits in many instances and more) and the implementation of the meme object can be very long.

\section{Reproduction}

The second stage of "memization" in the virtual information environment is distribution by copying and duplication. Most often, the spread and rapid growth of an enthusiastic audience (people who saw this element of information) is due to the copying of the primary precedent by a person who uses the fame / recognition on the Internet (repeater). Hence, the disappointing conclusion - absolutely anything can turn into a meme, even what should not turn into it. And at this stage, too, the dominant role of coincidence is noticeable.

In terms of architectural public space, replication conducts much slower. With the advent of a new meme in space (exposing it to the general public), first convinced of its effectiveness (that the content embedded in it is justified and is functional). For example, if you take a bollard as a meme, you first certify that it fulfills its mission in space (restricts the passage of cars) and other people without 
explanation understand its function. Thus, replication does not depend on one person, but on the overall impression in relation to the object. Of course, the budget for creating copies of this object plays an important role in delaying duplication.

Fig. 2. New York, San Francisco use circles to help social distance efforts in parks. A precedent phenomenon. Source: AP Photo/Kathy Willens

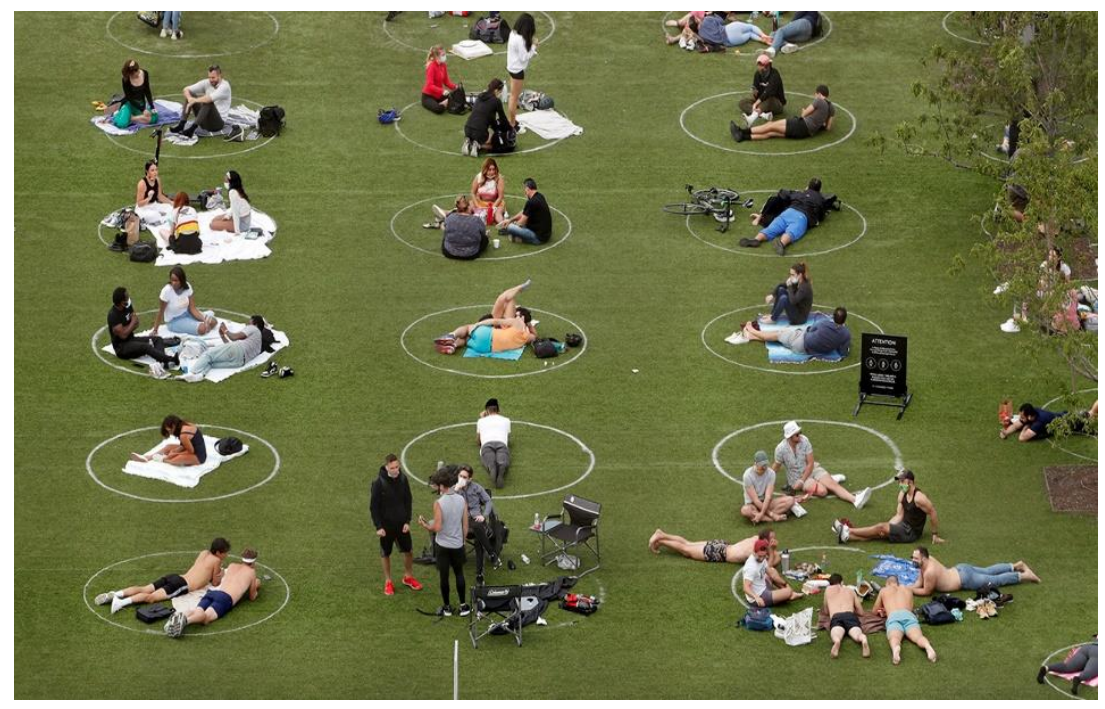

At this stage of comparative analysis, virtual and physical spaces can intersect. This happens when an object in physical space is photographed / videotaped / scanned and information about it is disseminated much faster over the Internet.

\section{Transmission and storage of cultural information}

Memes are a part of folklore, before the advent of the Internet they lived in a shape of anecdotes, jokes and lyrics that were quickly and easily memorized and transmitted. Very often the question arises: are memes, in general, arise externally, as a reaction to processes in culture and processing / interpretation of them in their own way, or are memes - a product of intracerebral activity? Be that as it may, based on the first point of analysis of the "precedent phenomenon", it becomes obvious, that each meme is the bearer of the history, that created it.

Architectural memes, as opposed to virtual ones, tend to show more the history of human progress or new activities that need to be introduced into the public space due to new social, cultural, economic or other circumstances. In many cases, the reason for the involvement of the meme in space is the non-functional use of the territory or the lack of certain minimum sonorities. An internet meme usually has a comedic, shocking, provocative tone or expresses a display of black humor in relation to the info drive - so the information is more likely to be touching and will be replicated faster. Recently, the architectural meme is also increasingly attracting attention due to its dynamism in the entire static space of the city (moving objects in space with which a passerby interacts). The reason for this phenomenon lies in the general acceleration of the pace of life in the city, the acceleration of information exchange and in another level of emotional vulnerability of city residents.

\section{Special information}

Not every piece of information can become a meme. Only information that is relevant to a lot of people, more often, on topical issues with comic, painful, aggressive, absurd backbone, provoking the desire to share content (most often, it is a combination of photo / video with a sharp inscription) takes part in the memization process. The degree of universality of the meme is also very important, so that it will be able to become relevant in any situation. 
In the case of an architectural meme, a functional / useful element, primarily a visually appealing element / in some cases, a visually repulsive element, that is easy to remember, is involved in the further dissemination process. The meme can also be a unique author's work, which is mostly modified in a different context and space (imitation, plagiarism). The rule remains inviolable: an architectural meme will not be a physical object of space without a strong emotional response from consumers of this space.

\section{Virality}

In the context of viral information carried by a meme, a comparison with lines of a song that cannot be thrown out of one's head is the best. There are several recipes for Internet memes that help to promote a unit of information and create a resonance on the Internet. First, it is content (info material) that in any way attracts the attention of the audience. An attractive title, an intriguing text part, a visual component (info graphics, images, animation, video) and provoking emotions play a role in attracting attention. Playing on emotions can be both positive and negative, because the negative unites people faster and much stronger. The second recipe is storytelling, and best of all, not an impersonal story - but on behalf of a particular person. In addition, the audience's attention should be kept. New details, intrigue, advertising and creating conflicts are helpful for this task.

Virality for an architectural meme works in a similar way. The element of space, scandalous even at the project stage, is guaranteed to attract the attention of citizens and will be popular in the first month or two after implementation. To keep the excitement and start replication, you need to nurture the fame gained from the outside - to involve famous people in the story of the object, to involve an element of space in advertising campaigns, filming, reporting, competitions, publications in relevant online publications. Virality also depends on the very characteristics of the object: does it evoke strong emotions and trust when interacting with it, or does it reflect its essence through simple archetypes? Archetypes are images, certain characteristics and qualities, that are engraved in the human psyche and seem universal, so we immediately understand them at the level of intuition.

\section{Public place of "origin"}

Internet memes are an exclusively public phenomenon from the very beginning. They cannot exist without carriers (people) and, otherwise, die. In general, memes are a parasitic and non-independent unit of information with a constant need for energy recharging. Moreover, the energy should not come from one person (author), but affect as many people as possible. Information, whether it is a post on social networks or a new type of street furniture in public space, needs discussion, controversy and scandal to prolong its life and spread as far as possible.

From an architectural point of view, this is compared to the reaction of the public to the discovery of a new small architectural form. The more people come into contact with a given object of space (visual, tactile), the more there is a transformation of the original idea / message of the author. The object becomes overgrown with folk names, associations, descriptions and meanings. Over time and changing of cultural paradigms, this object can become a tourist attraction, replicated in other circumstances / places in modified form, and completely lose its artistic value. Thereby, the fate of the architectural meme is entirely in the hands of public approval / condemnation or its gradual loss of significance.

\section{Degree of locality}

An Internet meme will cover only a specific part of the Internet in two circumstances. The first is the local dissemination of information "only for your peers" in a closed public, forum, chat, pages on social networks (access to the website is provided by the administrator to a limited number of people). But this is not a completely inaccessible virtual space, only a temporarily privatized part of the "surface network" - movement on it can be easily tracked. For complete anonymity, there is a deeper level so-called "deep web". Deep web pages cannot be found in regular search because they are not indexed by search engines. There is also a darknet - a closed network of deep internet. There can be many such darknets (really closed virtual spaces) and each one needs special access. Internet memes distributed in such an environment are purely local and even personal. 
By analogy with the Internet, there are public spaces with limited access (state ownership, private enterprise, gated communities, educational institutions, etc.). Each element of such a public space is an indicator of the privilege and allocation of people who have the key to this territory. The meaning of a certain element of such space will be better understood by a limited circle of people and, accordingly, will be slower to copy.

\section{Narrowing the target audience of the meme}

Deliberate narrowing of the availability of Internet memes occurs in a group of people united by one profession, interests, occupation, subculture, where there is a special vocabulary / visual stock and specific topics of discussion that will be incomprehensible to the general public (eg IT professionals, health professionals, rockers or electronic music lovers). Therefore, memes will cause emotional reactions only in specific people with a limitation of understanding for others.

Narrowing the audience for an architectural meme is mainly not textual, but visual-tactile or sound. That means the exclusion of entire public spaces or their elements from the accessibility for the average city dweller. For example, the potential danger of certain spaces is related either to their physical characteristics or to the possibility of encountering deviant individuals or aggressive animals - people bypass these spaces in advance. Another exception may be the impossibility or limited access to certain places or types of mobility, due to the actions of third parties (if it is the territory of public or private organizations, local authorities, educational institutions). Space and meme elements in it are also inaccessible if the needs of people with disabilities, children, the elderly and other categories of the population who are temporarily not fully physically capable are respected.

\section{Short life}

The digital life of an internet meme is usually short term. There is a moment of chaotic, unpredictable origin, the growth of the covered audience, the peak moment of popularity and rapid decline, by displacing this meme by newer and relevant. After the digital "death", the meme continues to carry information about a particular period of its popularity, but in very narrow circles. The use of this meme in everyday, especially between youth virtual communication shows a person as outdated and one, who does not follow the changes in the Internet environment.

The situation is more complicated with an architectural meme. Firstly, the rate of dissemination of information is very different in virtual and real spaces, and therefore the phases of "new - relevant bored / out of fashion - dying" are very stretched in time for physical space. Often, the last phase of a meme's life may not be at all. This applies to elements of space that were invented / originated historically many centuries ago and do not lose their relevance to this day. For example, it's stairs and benches. The objects have undergone many transformations and additions, but the main ideological backbone and the main function remains - their number and degree of dissemination in public spaces is very high. For such memes there are no restrictions on age, gender, national, cultural factor, because their relevance is acceptable to absolutely everyone and regardless of the situation.

\section{Equivalence of cultural baggage}

For the popularity of any object its manifestation (public recognition) by the potential public is very important. And to be recognized, the element, first of all, must be clear, universal and intuitive. Internet memes, as a relatively young cultural phenomenon, have as their target audience mainly people who were born either during the transition to Internet technology or when these technologies have become commonplace. That is, for an instant understanding of a meme, it is not important for a person to have a rich life experience, but to be aware of the latest news / cultural and social events / movie premieres. In some cases, memes convey the essence of the information phenomenon so briefly and meaningfully that it is a substitute for standard news resources (news releases, newspaper articles or online publications). The level of coding of information in the meme must also coincide with the mental maturity of the person, so there are memes that are conventionally divided for different age groups. 
A similar rule applies in architecture. Memes that have developed historically and have a long period of activity are perceived properly and do not raise questions. Newer memes that have come into use relatively recently (technologically sophisticated) are instantly understandable to people with a certain level of mental development (who are actively included in the cultural context), while for others at first contact they can cause surprise and even confusion.

\section{Simplification}

The main task of any Internet meme is to broadcast information, news, emotional message in a simplified, understandable form to cover the largest possible audience. By structural type there are twosyllable, character, syntactic, situational and comparative memes. This division is due to the selection of the part of the meme that carries the main semantic load (be it one image, phrase, comics or several images).

Accordingly, in architecture, each meme has a main semantic part and an auxiliary one. A summary, signal, sign, or specific stimulus may be hidden in the shape, outline, texture, color, or other defining characteristic of the object. For example, tactile ground warning signs on sidewalks both in color and texture signal their importance in space and possible actions in relation to them.

\section{CONCLUSION}

Having considered a brief history of the formation and development of the concept of a meme, it becomes clear that our daily life consists of all objects-symbols that carry a certain meaning and in architecture / in public spaces of cities there are their own memes. As a result of the comparative analysis, it became clear that architectural memes have similar characteristics to Internet memes. The big difference lies in the timing of the emergence of Internet memes (late 90s and early 2000s) and architectural memes (they arose with the inception of architecture itself) and the experience / information that during this time was absorbed into the concept of the meme itself. In the context of such conclusions, it is valuable to identify the main types of architectural memes, the qualities that transform them into such, the context and causes of occurrence, transformation and even death. This topic is original and relevant for modern architectural research.

\section{BIBLIOGRAPHY}

Bataille G. Une liberté souveraine: Textes et entretiens, (articles, book reviews and interviews not included in Oeuvres Complètes, Michel Surya Ed.) 2004

Blackmore S. The Meme Machine (1st ed.). Oxford University Press. 1999.

Dancy R. Plato's Introduction of Forms. Cambridge: Cambridge University Press. 2004

Dawkins R. The Selfish Gene (2nd ed.). United Kingdom: Oxford University Press.1989

Deleuze G. "Intellectuals and Power: A Discussion Between Gilles Deleuze and Michel Foucault". TELOS 16. New York: Telos Press (reprinted in L'île déserte et autres textes / Desert Islands and Other Texts). 1973

Lumsden C. J. Genes, Mind and Culture: The Coevolutionary Process / C. J. Lumsden, E. O. Wilson. - Cambridge (MA), 1981.

Rushkoff D. Team Human. W. W. Norton Company. 2019

Wilson E. Genes, Mind and Culture: The Coevolutionary Process, Harvard University Press. 1981

\section{AUTHOR'S NOTE}

Holovatiuk Alina, architect, master of architecture, PhD student at the Department of Information technologies in Architecture of the Kyiv National university of Construction and Architecture. Alina Holovatiuk conducts her research on memes in Architecture for acquiring Ph.D. degree.

Contact | Kontakt: Deppyio1212@gmail.com 